\title{
Íleo biliar en adulto joven. Reporte de un caso
}

\author{
Biliary ileus in a young adult. A case report \\ Yady Vanessa Hurtado1* orcid.org/0000-0001-5665-0203 \\ Hanier Agudelo² orcid.org/0000-0001-9967-6139 \\ Ángela María Merchán-Galvis² orcid.org/0000-0003-2375-5513
}

1 Unidad de Cuidados Intensivos, Hospital Universitario San José. Popayán, Colombia

2 Universidad del Cauca. Popayán, Colombia

\section{Resumen}

Introducción: El íleo biliar se define como una obstrucción intestinal por impactación de un cálculo biliar a cualquier nivel, sucede por presencia de una fistula bilio-digestiva y es usual en personas mayores de 60 años, principalmente en mujeres. objetivo: Describir un caso de íleo biliar en un paciente masculino adulto medio, tratado con enterolitotomia más colecistectomía. Presentación del caso: Paciente masculino de 41 años, sin antecedentes patológicos conocidos, con diagnóstico de íleo biliar, quién recibió manejo quirúrgico con enterolitotomia más colecistectomía en el mismo tiempo quirúrgico, con posteriores reintervenciones y manejo en unidad de cuidado intensivo, teniendo evolución favorable y egreso hospitalario. Conclusión: Este caso de obstrucción intestinal en paciente sin antecedentes quirúrgicos abdominales y con edad de presentación atípica para íleo biliar, representa un reto para el diagnóstico clínico y su correspondiente manejo quirúrgico.

Palabras clave: Colelitiasis; ileus; obstrucción intestinal; fistula biliar (Fuente: DeCS, Bireme).

\begin{abstract}
Introduction: Biliary ileus is defined as an intestinal obstruction caused by gallstones. It is due to the presence of a biledigestive fistula and is common in people older than 60 years of age, mainly in women. Objective: To describe a case of biliary ileus in an average adult male patient treated with enterolithotomy and cholecystectomy. Presentation of the case: A 41-year-old male patient, with a diagnosis of biliary ileus and no known pathological history, who underwent surgical management with enterolithotomy and cholecystectomy at the same surgical time. He had subsequent surgical interventions and management in an intensive care unit, having a favorable outcome and hospital discharge. Conclusion: This case of intestinal obstruction in a patient with no abdominal surgical history and an atypical age presentation represents a challenge for clinical diagnosis of biliary ileus and its corresponding surgical management.
\end{abstract}

Keywords: Cholelithiasis; ileus; intestinal obstruction; biliary fistula (Source: DeCS, Bireme).

\footnotetext{
*Autor de correspondencia

Yady Vanessa Hurtado

e-mail: vanessa_hurtado@hotmail.es
} 


\section{Introducción}

El íleo biliar es una condición poco común, descrita por primera vez por Erasmus Bartholin en 1654(1). Usualmente, se presenta en personas mayores de 60 años, predomina en mujeres(2) y es responsable de alrededor del 1\%-4\% de obstrucciones intestinales mecánicas(3). Se define como una oclusión digestiva mecánica debida a la obstrucción intraluminal por un cálculo biliar como complicación rara de la colelitiasis ${ }^{(2)}$. Ocurre por migración de uno o varios cálculos a través de una fístula bilio-digestiva ${ }^{(4)}$, definida como una comunicación entre la vesícula biliar o la vía biliar y algún tramo del intestino(5) que sucede de manera espontánea(6); permitiendo que los cálculos progresen a través del tubo digestivo hasta impactarse a cualquier nivel(7), por deterioro de la función peristáltica gástrica o intestinal(2). La piedra angular del tratamiento es la cirugía; sin embargo, el enfoque quirúrgico óptimo esta aun en debate(2).

\section{Descripción del caso}

En junio de 2017 un constructor de 41 años procedente de área urbana, sin antecedentes de importancia; consultó por dolor abdominal tipo cólico en epigastrio y en marco colónico, generalizado, asociado a emesis posprandial alimentaria, posteriormente fecaloide y ausencia de deposiciones, con una semana de evolución.

Al ingreso se encuentra en buena condición general, sin signos de dificultad respiratoria, no lucía álgico, frecuencia cardiaca 80 lat/min, presión arterial $90 / 60 \mathrm{mmHg}$, frecuencia respiratoria $20 \mathrm{resp} / \mathrm{min}$, temperatura $36^{\circ} \mathrm{C}$, saturación de oxigeno $96 \%$ con fracción inspiradora de oxigeno (FiO2) al 21\%. Mucosas semisecas, abdomen con peristaltismo presente y disminuido, defensa muscular a la palpación, con dolor en mesogastrio y marco cólico, sin signos de irritación peritoneal, al tacto rectal esfínter anal tónico y ampolla rectal vacía.

Con impresión diagnóstica de obstrucción intestinal se inició manejo con líquidos endovenosos, protección gástrica, analgésicos, antiespasmódico y antiemético. Se realizó paso de sonda nasogástrica y se obtuvo abundante material fecaloide. La serie radiológica de abdomen evidenció niveles hidroaéreos y ausencia de gas distal (figura 1).

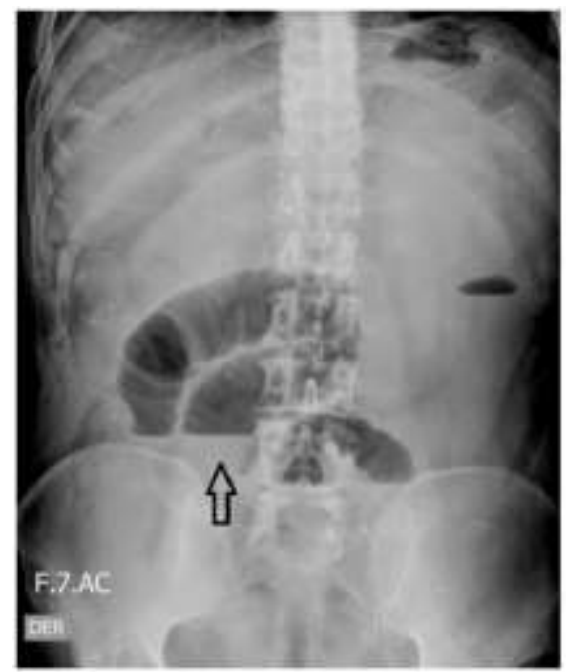

Figura 1. Radiografía simple de abdomen donde se observan niveles hidroaéreos (flecha), indicando obstrucción intestinal

La tomografía de abdomen total simple y contrastada reportó "Vesícula biliar no visualizada, en su reemplazo se observan cambios en la densidad del hilio hepático y lecho vesicular, con colecciones de aire. A nivel de la válvula ileocecal reducción importante de calibre, no se observa cuerpo extraño ni masa, tampoco alteraciones de la grasa peri-cecal. Considerar engrosamiento o efecto estenótico parcial a nivel de válvula ileocecal que condiciona obstrucción intestinal (solo intestino delgado). Se observa distensión marcada de todas las asas delgadas desde el duodeno hasta el íleon distal" (figuras 2 y 3 ).

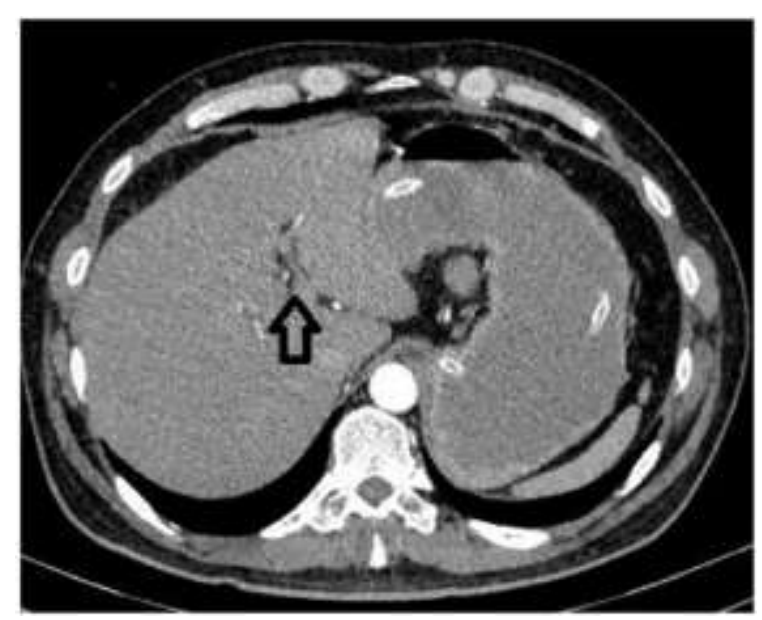

Figura 2. Tomografía axial computarizada de abdomen que evidencia neumobilia en el sitio donde anatómicamente debería estar la vesícula. 


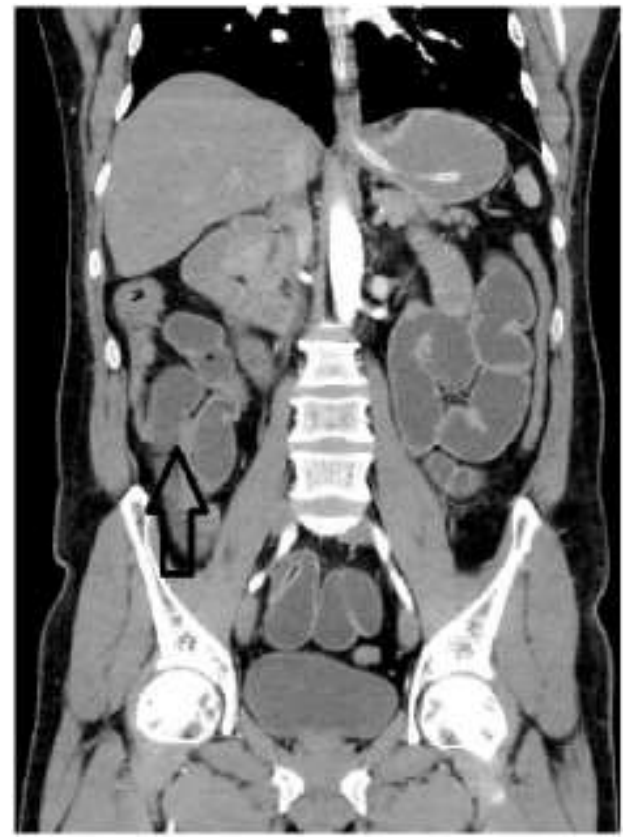

Figura 3. Tomografía axial computarizada de abdomen con efecto estenótico parcial a nivel de la válvula ileocecal que condiciona obstrucción intestinal.

Ante la sospecha de íleo biliar vs. efecto de masa en íleon distal y por la reducción de peristaltismo, distensión abdominal, dolor en los cuatro cuadrantes, persistencia de emesis fecaloide y producción abundante de este material por sonda nasogástrica; se llevó a manejo quirúrgico por laparotomía exploratoria, cuyos hallazgos fueron: asas intestinales distendidas, íleo de aproximadamente $1.600 \mathrm{cc}$, dilatación marcada de válvula ileocecal conteniendo cálculo biliar de aproximadamente $6 \times 2 \mathrm{~cm}$. Plastrón la vesícula que además está atrófica y con fístula hacia la segunda porción del duodeno.

Se realizó enterotomía en íleon distal y extracción del cálculo, drenaje parcial de íleo y enterorrafia. Se efectuó liberación del plastrón, colecistectomía y rafia del defecto de la pared anterior en la segunda porción del duodeno. Debido a la dificultad en el avance convencional se procedió a realizar una gastrotomía para paso de sonda nasoyeyunal, se realizó gastrorrafia y drenaje retrógrado de íleo intestinal. Finalmente se cerró pared por planos.

Por adecuada evolución se inició nutrición enteral a los tres días de posquirúrgico. En el cuarto día posquirúrgico presentó signos de respuesta inflamatoria sistémica dados por taquicardia con frecuencia cardiaca $104 \mathrm{lat} / \mathrm{min}$, leucocitosis de $16200 \mathrm{leu} / \mu \mathrm{L}$ y neutrofilia de $91 \%$, dolor abdominal generalizado a la palpación, signo positivo de Blumberg, herida quirúrgica con eritema y dehiscencia en tercio superior con salida de material fecaloide. Se suspendió nutrición enteral y se inició antibiótico de amplio espectro, fue llevado a cirugía el quinto día de posoperatorio, para descartar infección del sitio operatorio, colección intraabdominal o dehiscencia de rafias gastrointestinales.

Los hallazgos en el segundo tiempo quirúrgico feuron: herida quirúrgica con salida de material purulento, colección fétida en pared abdominal, peritonitis purulenta generalizada, síndrome adherencial severo viscero-visceral y omental, colección subfrénica derecha de 300 cc, perforación duodenal en primera y segunda porciones con dehiscencia de sutura previa en un $70 \%$, con bordes necróticos. Gastrorrafia y enterorrafia en íleon íntegras. Se realizó retiro de sutura previa, drenaje de colección de pared abdominal, liberación de adherencias, drenaje de peritonitis de cuatro cuadrantes y omentectomía total.

A continuación, se realizó maniobra de Kocher explorando la vía biliar, pero no fue posible identificación clara del colédoco distal y ampolla de Váter; se evidenció bilis retrógrada a perforación duodenal, vena porta y cava íntegras. Se procedió a gastrectomía subtotal a nivel de antro. En duodeno se liberó primera porción y se extrajo para estudio patológico, posteriormente se efectuó rafia del muñón duodenal con doble sutura invaginante y se realizó prueba de fuga. Después, se ascendió asa yeyunal con anastomosis latero-lateral en cara anterior del estómago, tipo Billroth II, empaquetamiento con dos compresas, se dejó viaflex libre en cavidad y viaflex a piel. El sangrado fue cuantificado en aproximadamente $1.000 \mathrm{cc}$.

Posteriormente el paciente requirió manejo en la unidad de cuidado intensivo (UCI), al ingreso se encontraron los siguientes signos vitales: frecuencia cardiaca 103 lat/min, presión arterial 85/45 mmHg, frecuencia respiratoria $15 \mathrm{resp} / \mathrm{min}$, temperatura $36^{\circ} \mathrm{C}$, bajo sedo-analgesia, monitoria hemodinámica invasiva, ventilación mecánica en modo volumen control, goteo de vasoactivo, con abdomen abierto, antibiótico de amplio espectro, terapia anti fúngica anticipada y nutrición parenteral total. Por adecuada evolución el segundo día de manejo en UCI fue extubado y luego se retiró el soporte vasoactivo. 
El paciente presentaba abdomen abierto, empaquetamiento con compresas y se documentó presencia de líquido de aspecto biliar sobre asas por lo cual fue llevado a tercer tiempo quirúrgico a las 72 horas de la intervención previa, encontrando peritonitis de cuatro cuadrantes teñida de bilis, membranas fibrinoides sobre intestino delgado, adherencias interasas laxas, anastomosis gastroyeyunal y muñón duodenal indemnes y sin filtraciones y se evidenció fuga desde conducto cístico. Se realizó drenaje de peritonitis, revisión de anastomosis y muñón duodenal, rafia de muñón del cístico, lavado de cavidad, colocación de dren custodio, viaflex libre y viaflex a piel; regresó a UCI para continuar manejo.

Paciente quien continua con abdomen abierto, hay drenaje de material seropurulento por dren custodio y presencia de bilioperitoneo a través de viaflex; al cuarto día de la última intervención con respuesta inflamatoria sistémica dada por leucocitosis de $16.700 \mathrm{leu} / \mu \mathrm{L}$, neutrofilia de $86 \%$ y proteína c reactiva (PCR) de $14 \mathrm{mg} / \mathrm{L}$; por lo cual se hizo necesario un cuarto tiempo quirúrgico donde se encontró zona de filtración de bilis entre el lecho hepático y muñón duodenal, de $3 \mathrm{~mm}$. Se practicó rafia de la zona de filtración en dos planos transfixiantes con control de la misma, no se identificaron otras áreas de fuga.

Durante la vigilancia, hubo persistencia de peritonitis de características biliares, visualizada a través el viaflex; por lo tanto, el paciente fue llevado a quinto tiempo quirúrgico, en este momento cumplía 15 días desde el ingreso hospitalario y 2 días desde la última intervención. Se halló peritonitis biliar no purulenta, fuga de bilis hacia el lecho vesicular sobre el parénquima hepático, se realizó drenaje de peritonitis, lavado de cavidad y se dejó fistula orientada con drenes abdominales \#2, continua con abdomen abierto y viaflex en piel.

Se mantuvo en seguimiento para orientación de la fistula y al continuar con abdomen abierto se realizó programación quirúrgica para cierre de cavidad con talla de colgajos, este sexto y último procedimiento se realizó al día 21 desde el ingreso y 7 días después de la cirugía previa.

Por mejoría clínica se inició alimentación liquida vía oral, la cual toleró adecuadamente y se progresó, presentó control del dolor, drenes abdominales con escasa producción biliosa, resolución de sepsis abdominal, sin nuevo requerimiento de intervención quirúrgica y fue egresado después de 31 días de hospitalización. En consulta ambulatoria se retiraron drenes y puntos de sutura de la herida quirúrgica; en el control a los 3 meses se documentó evolución satisfactoria y presencia de hernia ventral.

\section{Consideraciones éticas}

Este trabajo contó con el aval del comité de ética del Hospital Universitario San José de Popayán para la publicación del caso y tuvo en cuenta las normas vigentes sobre investigación en seres humanos, contempladas en la Declaración de Helsinki de la Asociación Médica Mundial y la Resolución 8430 de 1993 del Ministerio de Salud de Colombia. De igual manera se obtuvo el consentimiento informado del paciente para la revisión de su historia clínica y publicación de sus imágenes diagnósticas.

\section{Discusión}

El íleo biliar se ha convertido en una entidad de difícil diagnóstico pre-quirúrgico, pues en la mayoría de los casos se presenta como una obstrucción intestinal(8). Se considera una condición muy poco frecuente( ${ }^{(4)}$, prevalente en el sexo femenino, así como en mayores de 60 años y asociada a una alta morbimortalidad(2); lo anterior, contrasta con nuestro paciente que era hombre, de un grupo de edad por debajo de lo citado y sin factores de riesgo para patología biliar.

Corresponde a una complicación de la litiasis vesicular en la cual se produce una obstrucción mecánica del tracto gastrointestinal por uno o más cálculos biliares grandes en su luz ${ }^{(9)}$. El paso de un cálculo hacia el lumen intestinal se da a través de una fístula(10), la cual surge cuando hay episodios recurrentes de colecistitis aguda, provocando gran inflamación y adherencias entre la vesícula y el tracto gastrointestinal(11). La fístula más frecuente es la colecisto-duodenal, con cerca del $85 \%$ de los casos(12); otras localizaciones menos usuales son las fistulas que involucran al estómago y el colon(13).

El lugar de impactación depende del tamaño del cálculo y del diámetro de la luz del tubo digestivo(14). Una vez el cálculo se ubica en la luz intestinal puede generar obstrucción a cualquier nivel, el lugar más habitual es el íleon terminal (70\%), seguido por yeyuno - íleon proximal (25\%) y duodeno (5\%)(7). Los cálculos deben medir al menos $2 \mathrm{~cm}$ para ser 
causantes de obstrucción(12). Resulta difícil que cálculos menores a 2-2,5 cm generen oclusión, a menos que haya alteración en la dinámica intestinal o disminución de la luz ya sea debida a una condición benigna o maligna no diagnosticada(7). Cuando se obstruye el íleon terminal se presenta el síndrome de Barnard(12), en los casos en que el cálculo se ubica en el duodeno se produce el síndrome de Bouveret(12), menos del $5 \%$ de los pacientes presentan obstrucción en colon(12).

Respecto a la fístula más usualmente reportada en la literatura y el sitio más frecuente de obstrucción, se evidencia coincidencia, en ambos aspectos, con los hallazgos del paciente descrito. Es relevante anotar que el tamaño del cálculo hallado en el paciente $(6 \mathrm{~cm})$, supera por varios centímetros el tamaño mínimo requerido para ser causante de obstrucción según la literatura consultada.

La sintomatología frecuentemente referida, es el dolor abdominal tipo cólico inicialmente difuso, que después se ubica en hipocondrio derecho y puede extenderse a fosa iliaca derecha e hipogastrio(15). Se asocia a náuseas y vómito gastrobiliar persistente, distensión abdominal, ausencia de peristalsis, constipación, desequilibrio de líquidos(12) y puede presentar signos de irritación peritoneal(15).

El examen físico y las pruebas de laboratorio no señalan la causa específica de la obstrucción(12), por esto se sospecha íleo biliar en un paciente de edad avanzada con la triada de Mordor (antecedente de cálculo biliar, signos de colecistitis aguda y aparición repentina de obstrucción intestinal)(11).

En el cuadro clínico, hubo concordancia con lo reportado, al presentarse como una obstrucción intestinal(8), pero no fue posible determinar la causa exacta de manera pre-quirúrgica(12). Nuestro paciente requirió larga observación, estudios de laboratorio e imagen para lograr un enfoque diagnóstico más acertado. Las ayudas diagnósticas incluyen pruebas de laboratorio donde se puede encontrar leucocitosis, función hepática alterada, signos de deshidratación y en algunos casos desequilibrio electrolítico(12).

En cuanto a los estudios de imagen, se inicia con la toma de radiografías de abdomen de pie y en decúbito supino, el criterio diagnóstico para íleo biliar es la triada de Rigler: presencia de cálculos radio-opacos, neumobilia y distensión de asas intestinales(16). La aparición de dos de estos tres signos establece el diagnóstico; si se documenta el cambio de posición de un cálculo respecto a una radiografía previa, se configura la tétrada de Rigler(11).

El estudio ultrasonográfico aporta datos de la condición de la vesícula y la vía biliar, la presencia de fístula bilio-entérica, neumobilia o cálculos en la luz intestinal(7). También puede informar sobre dilatación de asas intestinales y líquido libre en cavidad abdominal; sin embargo, tiene baja sensibilidad debido a la interposición de gas(17).

La Tomografía computarizada (TAC) abdominal, permite evidenciar la ubicación del cálculo y de la obstrucción intestinal(8), visualizar neumobilia, neumocolecisto, fístula bilio-entérica, niveles hidroaéreos y liquido libre en cavidad(17,18), también permite identificar el tamaño del cálculo(11). La tomografía se considera el patrón de oro para el diagnóstico de íleo biliar(2) y si se usa contraste arroja una sensibilidad mayor al $90 \%{ }^{(19)}$. En las imágenes del paciente, la TAC de abdomen mostró aire en el lugar de la vesícula biliar, así como distensión de asas y obstrucción a nivel de la válvula ileocecal, lo cual orientó la sospecha diagnóstica hacia íleo biliar con la consecuente intervención quirúrgica que llevó a la resolución de su obstrucción.

El tratamiento quirúrgico se realiza como resultado del diagnóstico preoperatorio o por hallazgo intraoperatorio(12), su objetivo principal es el alivio inmediato de la obstrucción intestinal al eliminar el cálculo biliar(20); en el caso relatado el primer tiempo quirúrgico incluyó, entre otros, enterotomía para extracción del cálculo y colecistectomía.

Se han descrito varias técnicas para el abordaje, aunque no hay consenso sobre la elección del procedimiento quirúrgico ${ }^{(9)}$. Las opciones de manejo se han dividido en dos enfoques básicos. La primera alternativa es realizar enterolitotomía solamente, con o sin colecistectomía diferida(10); esta técnica se lleva a cabo con mayor frecuencia que otras, evitando cirugías más prolongadas o complicadas(12). La segunda es cirugía de una etapa, implica una combinación de enterolitotomía y cirugía biliar definitiva con colecistectomía y reparación de fístula(10), como ocurrió con el paciente del caso presentado. 
El caso descrito, a pesar de haber requerido múltiples intervenciones, tuvo un desenlace favorable en su evolución, se logró resolución de la obstrucción intestinal, así como recuperación de la funcionalidad del tracto digestivo, mejoría de la sepsis y posterior alta hospitalaria.

\section{Conclusión}

Este caso de obstrucción intestinal en paciente sin antecedentes quirúrgicos abdominales y con edad de presentación atípica para íleo biliar, representa un reto para el diagnóstico clínico y su correspondiente manejo quirúrgico.

Conflicto de intereses: Los autores negamos la existencia de algún conflicto de interés.

\section{Referencias}

1. García-Valenzuela SE, Trujillo-Bracamontes FS, QuinteroGarcía B, Ríos-Beltrán JC, Valdez-Avilés D. Obstrucción intestinal secundaria a íleo biliar: reporte de dos casos. Rev Esp Med Quir. 2015;20:111-5. Disponible en: https://www.medigraphic.com/cgi-

bin/new/resumen.cgi?IDARTICULO=57219

2. Scuderi V, Adamo V, Naddeo M, Di Natale W, Boglione L, Cavalli S. Gallstone ileus: monocentric experience looking for the adequate approach. Updates Surg. 2018 Dec;70(4):503511. doi: 10.1007/s13304-017-0495-z. Epub 2017 Oct 10. PubMed PMID: 29019098.

3. De Monti M, Cestaro G, Alkayyali S, Galafassi J, Fasolini F. Gallstone ileus: A possible cause of bowel obstruction in the elderly population. Int J Surg Case Rep. 2018;43:18-20. doi: 10.1016/j.ijscr.2018.01.010. Epub 2018 Feb 4. PubMed. PMID: 29414501; PubMed Central PMCID: PMC5907688.

4. Salazar-Jiménez, M. I., Alvarado-Durán, J., Fermín-Contreras, M. R., Rivero-Yáñez, F., Lupian-Angulo, A. I., \& HerreraGonzález, A. (2018). Íleo biliar, revisión del manejo quirúrgico. Cirugia y cirujanos, 86(2), 182-6. doi: 10.24875/CIRU.M18000032

5. Aláez-Chillarón, A. B., Moreno-Manso, I., Martín-Vieira, F. J., Mojtar, M. F., \& Pérez-Merino, E. (2017). Gallstone ileus after endoscopic retrograde cholangiopancreatography. Cirugía y Cirujanos (English Edition), 85(2), 154-7. https://doi.org/10.1016/j.circen.2017.02.003

6. Aguilar-Espinosa F, Maza-Sánchez R, Vargas-Solís F, Guerrero-Martínez GA, Medina-Reyes JL, Flores-Quiroz PI. Cholecystoduodenal fistula, an infrequent complication of cholelithiasis: Our experience in its surgical management. Rev Gastroenterol Mex. 2017 Oct - Dec;82(4):287-95. https://doi.org/10.1016/j.rgmxen.2017.07.015

7. Marenco-de la Cuadra B, López-Ruiz JA, Tallón-Aguilar L, López-Pérez J, Oliva-Mompeán F. Íleo biliar colónico: una rara causa de obstrucción intestinal. Cir Cir 2017;85(5):4403. doi: 10.1016/j.circir.2016.05.016

8. Chacón, J. R., Castañeda, M. C. M., \& Mora, J. A. B. (2017). Íleo biliar, resolución quirúrgica en un solo tiempo: Evolución post-operatoria. Reporte de caso. Revista Médica de la
Universidad de Costa Rica, 11(2), 45-52. https://doi.org/10.15517/rmucr.v11i2.34578

9. Salamea, J. J., \& Salamea, P. A. (2017). Ileo biliar, reporte de caso clínico. Revista de la Facultad de Ciencias Médicas, 35(1), 85-9. Disponible en: https://publicaciones.ucuenca.edu.ec/ojs/index.php/medici na/article/view/1243

10. Martín-Pérez, J., Delgado-Plasencia, L., Bravo-Gutiérrez, A., Lorenzo-Rocha, N., Burillo-Putze, G., \& Medina-Arana, V. (2015). Enterolitotomía más colecistectomía precoz, una aplicación de cirugía de control de daños para pacientes con íleo biliar. Cirugía y Cirujanos, 83(2), 156-60. https://doi.org/10.1016/j.circir.2015.04.013

11. Beuran M, Ivanov I, Venter MD. Gallstone ileus-clinical and therapeutic aspects. J Med Life. 2010 Oct-Dec;3(4):365-71. Disponible

en: https://www.ncbi.nlm.nih.gov/pmc/articles/PMC3019077/ pdf/JMedLife-03-365.pdf

12. Ploneda-Valencia CF, Gallo-Morales M, Rinchon C, NavarroMuñiz E, Bautista-López CA, de la Cerda-Trujillo LF, ReaAzpeitia LA, et al. El íleo biliar: una revisión de la literatura médica. Rev. Fac. Med 2017;82(3):248-54. https://doi.org/10.1016/j.rgmx.2016.07.006

13. Sánchez-Pérez, E. A., Álvarez-Álvarez, S., Madrigal-Téllez, M. A., Gutiérrez-Uvalle, G. E., Ramírez-Velásquez, J. E., \& Hurtado-López, L. M. (2017). Gallstone ileus, experience in the Dr. Eduardo Liceaga General Hospital of Mexico. Cirugía y Cirujanos (English Edition), 85(2), 114-120. https://doi.org/10.1016/j.circen.2017.02.008

14. Rojas-Rojas DJ, Martínez-Ordaz JL, Romero-Hernández T. Íleo biliar, causa poco frecuente de obstrucción intestinal. Rev Gastro Méx. 2014;79(3):211-3. https://doi.org/10.1016/j.rgmx.2014.05.008

15. Ayantunde A, Agrawal A. Gallstone ileus: diagnosis and management. World J Surg. 2007;31(6):1292-7. doi: 10.1007/s00268-007-9011-9

16. Brandariz-Gil Lorena, Fernández-de-Miguel Tamara, Perea José. Rigler triad in gallstone ileus. Rev. esp. enferm. dig. [Internet]. 2016 Sep [citado 2019 Dic 18] ; 108( 9): 581$581 . \quad$ Disponible en: http://scielo.isciii.es/scielo.php?script=sci_arttext\&pid=S11 30-01082016000900013\&lng=es.

17. Bernal A, Gómez Velásquez MA, Molano F. Íleo biliar: reporte de un caso. Rev. colomb. Radiol. 2008;19(3):2476-8.

18. Chang L, Chang M, Chang HM, Chang AI, Chang F. Clinical and radiological diagnosis of gallstone ileus: a mini review. Emerg Radiol. 2018 Apr;25(2):189-196. doi: 10.1007/s10140-0171568-5. Epub 2017 Nov 16. Review. PubMed PMID: 29147883; PubMed Central PMCID: PMC5849656.

19. Ibrarullah M, Samantaray PK, Meher S, Bruno WH. Gallstone ileus of upper gastrointestinal tract: A case series. Indian J Gastroenterol. 2019 Jun;38(3):273-275. doi: 10.1007/s12664-019-00954-7. Epub 2019 Jun 12. PubMed PMID: 31190230.

20. Hussain J, Alrashed AM, Alkhadher T, Wood S, Behbehani AD, Termos S. Gall stone ileus: Unfamiliar cause of bowel obstruction. Case report and literatura review. Int J Surg Case Rep. 2018;49:44-50. doi: 10.1016/j.ijscr.2018.06.010. pub 2018 Jun 25. PubMed PMID: 29960209; PubMed Central PMCID: PMC6039705. 